\section{POS1419 PREDICTIVE FACTORS FOR PULMONARY PROGRESSION IN PATIENTS WITH CONNECTIVE TISSUE DISEASE AND INTERSTITIAL LUNG DISEASE}

Y. H. Chiu ${ }^{1,2}$, J. Spierings ${ }^{1}$, P. De Jong ${ }^{3}$, F. Mohamed Hoesein ${ }^{3}$, J. M. Van Laar', M. Voortman ${ }^{4} .{ }^{1}$ University Medical Centre Utrecht, Department of Rheumatology and Clinical Immunology, Utrecht, Netherlands; ${ }^{2}$ National Defense Medical Center, Division of Rheumatology/Immunology/Allergy, Taipei, Taiwan, Republic of China: ${ }^{3}$ University Medical Centre Utrecht, Department of Radiology, Utrecht, Netherlands; ${ }^{4}$ University Medical Centre Utrecht, Department of Heart and Lung, Utrecht, Netherlands

Background: Interstitial lung disease (ILD) is associated with decreased quality of life and higher mortality risk in patients with connective tissue disease (CTD). Outcome and treatment response to immunosuppressive therapies is unpredictable, and therefore the management of CTD-ILD can be challenging.

Objectives: Our study aimed to identify clinical and imaging factors that are predictive for outcome in patients with CTD-ILD.

Methods: We performed a retrospective cohort study in patients with CTD-ILD who were treated in our centre between 2004 and 2018. Clinical, biochemical data as well as pulmonary function test (PFT) and high-resolution computed tomography (HRCT) results were recorded. Two experienced chest radiologists independently and blindly reviewed the HRCT's. When the two chest radiologists assessed the ILD pattern differently, a diagnosis was made by consultation of a third expert. The ILD patterns were classified as fibrotic or inflammatory. Overall survival and progressive fibrosing interstitial lung disease (PF-ILD, defined as a significant decline of PFT and HRCT) after two years of treatment were assessed using a Kaplan-Meier plot. Multivariable Cox regression was including for treatment, comorbidity, and age as variables. Factors with a $p$ value $<0.2$ in the univariate analysis were included in the multivariate analysis. The correlation between the variation of serum markers and PFT over-time was evaluated with Spearman's Rho

Results: In total, 150 patients with CTD-ILD were included, of which 53 (35.3\%) had systemic sclerosis, 19 (12.7\%) Sjogren's syndrome, 29 (19.3\%) inflammatory myopathy, $24(16 \%)$ rheumatoid arthritis, $5(3.3 \%)$ systemic lupus erythematosus, $4(2.7 \%)$ mixed connective tissue disease, and 16 $(10.7 \%)$ undifferentiated connective tissue disease patients. Median disease duration of CTD was 14 months (IQR 2-73) in patients with CTD diagnosis before ILD onset. The median follow-up duration was 40 months (IQR $27.3-60.8)$. Thirty $(20 \%)$ deaths occurred, in which the cause of death was a pulmonary infection in $6(4 \%)$ patients and a respiratory failure due to ILD in $10(6.7 \%)$ patients. PF-ILD occurred in $82(54.7 \%)$ patients, which was associated with poor overall survival (HR $3.03,95 \% \mathrm{Cl} 1.15-7.98$ ) (Figure 1). Age, smoking, and steroid usage were associated with increased mortality risk as well (Table 1). There was no dose-related effect of smoking on mortality.

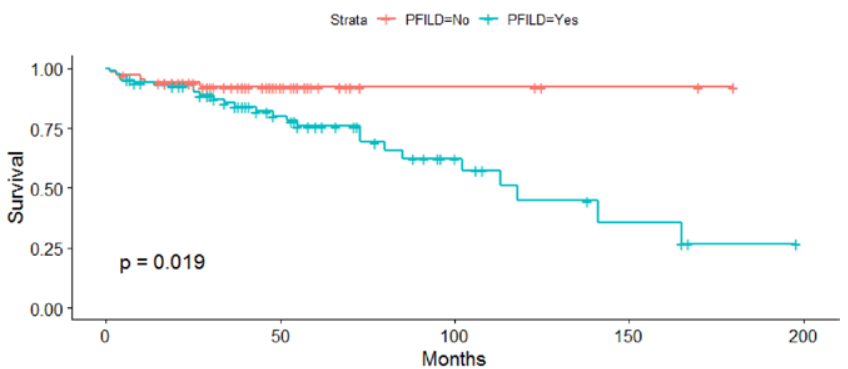

Figure 1. The Kaplan-Meier plot for progressive fibrosing interstitial lung diseases (PF-ILD). PF-ILF was defined as pulmonary function decline or high-resolution computed tomography progression after two years of treatment.

Inflammatory patterns on baseline HRCT were correlated with a lower risk of FVC decline than fibrotic patterns (OR $0.24,95 \% \mathrm{Cl} 0.09-0.64)$. The increase in CA15.3 level was associated with the decline in FVC (Rho -0.308, $p=0.037$ ). Besides, the elevation in CRP was associated with the reduction in FVC (Rho -0.302, $\mathrm{p}=0.006$ ) and DLCO (Rho $-0.268, \mathrm{p}=0.019$ ).

Conclusion: Our study identified several factors associated with outcomes. Age, smoking, and steroid treatment increased the risk of mortality in patient with CTD-ILD. Inflammatory HRCT pattern at baseline revealed a better pulmonary outcome than a fibrotic pattern. The patients having PF-ILD after two years of treatment showed a higher mortality risk.
Table 1. Multivariable Cox-regression for the clinical risk of mortality.

\begin{tabular}{lcccc}
\hline Clinical factor & Crude $\mathrm{HR}(95 \% \mathrm{Cl})$ & $\mathrm{P}$ & Adjusted HR $(95 \% \mathrm{Cl})$ & $\mathrm{p}$ \\
\hline Age & $1.11(1.06-1.15)$ & $1.7^{\star} 10^{-6}$ & $1.12(1.07-1.17)$ & $3.54^{*} 10^{-6}$ \\
Smoking & $1.64(0.79-3.43)$ & 0.187 & $2.53(1.11-5.78)$ & 0.028 \\
Congestive heart failure & $1.86(0.75-4.58)$ & 0.179 & $1.17(0.47-2.91)$ & 0.737 \\
MMF & $0.55(0.23-1.35)$ & 0.195 & $0.73(0.29-1.85)$ & 0.512 \\
Steroid & $4.37(1.67-11.45)$ & 0.003 & $4.96(1.84-13.40)$ & 0.002 \\
\hline
\end{tabular}

MMF, mycophenolate mofetil; HR, hazard ratio.

Acknowledgements: We want to thank Marieke Vianen for the support in data management, Lieke Wintermans and Lisa Hessels for collecting the clinical data Disclosure of Interests: None declared

DOI: 10.1136/annrheumdis-2021-eular.261

\section{POS1420 RISK OF SERIOUS INFECTIONS IN OFFSPRING EXPOSED IN UTERO TO USTEKINUMAB OR VEDOLIZUMAB}

J. Gorodensky ${ }^{1}$, S. Bernatsky ${ }^{1,2}$, W. Afif ${ }^{3}$, Y. St-Pierre ${ }^{2}$, K. Filion ${ }^{1,4}$, E. Vinet ${ }^{1,2}$. ${ }^{1}$ Mc Gill University, Epidemiology, Montréal, Canada; ${ }^{2}$ Research Institute of the McGill University Health Centre, CORE, Montréal, Canada; ${ }^{3} \mathrm{Mc}$ Gill University, Gastroenterology and Hepatology, Montreal, Canada; ${ }^{4}$ Lady Davis Institure for Medical Research, Center for Clinical Epidemiology, Montreal, Canada

Background: Ustekinumab, an IL-12/23 inhibitor, is indicated in adult patients with inflammatory bowel disease (IBD), psoriasis (PsO), and psoriatic arthritis (PsA) and vedolizumab, an $\alpha_{4} \beta_{7}$ receptor antagonist is indicated in IBD only. Both are monoclonal antibodies harbouring an Fc portion, which are actively transported across the placenta, often reaching higher fetal than maternal levels. As fetuses could be exposed to therapeutic or supra-therapeutic levels of these drugs, there are concerns that these agents could cause immunosuppression after birth. However, evidence is lacking.

Objectives: We compared the risk of serious infections in offspring exposed to ustekinumab, vedolizumab, tumour necrosis factor inhibitors (TNFi), and non-biologic immunosupressives versus offspring unexposed during pregnancy among women with IBD, PsO and/or PsA.

Methods: We conducted a retrospective cohort study using the US MarketScan database, an employment insurance database. We included live births (01/2011 12/2018) among women with PsO, PsA, and/or IBD. Drug exposure was defined as $\geq 1$ filled prescrip tion or infusion procedure code during pregnancy. In offspring, we evaluated serious infections within the first year of life as any single inpatient infection code. We performed multivariate analyses using logistic regression, adjusting for maternal age, co-morbidities, corticosteroid use, concomitant drug use, and preterm birth.

Results: We included 16,115 offspring born to 7,612 women with PsO/PsA, 8,315 with IBD, and 188 with PsO/PsA and IBD. A total of 52 offspring were exposed to ustekinumab, 43 to vedolizumab (including 7 to both TNFi and vedolizumab), 1,578 to TNFi, 1,857 to non-biologic immunosuppressives alone, and 12,585 unexposed to any drug. The percentage of serious infections in offspring exposed to ustekinumab was $3.9 \%$ (95\% $\mathrm{Cl} 0.5-13.9)$, versus $2.7 \%(95 \% \mathrm{Cl} 1.9$ 3.6) for TNFi, 2.3\% (95\% Cl 0.06-13.0) for vedolizumab and $2.6(95 \% \mathrm{Cl} 2.3-2.8)$ for those unexposed to any drug, though all estimates had wide, overlapping confidence intervals. Compared to children unexposed to any drug, there was a potential trend for increased risk with ustekinumab (OR 1.54, 0.37-6.45), but Cls were wide and included the null. For those exposed to vedolizumab (OR 0.82 0.15-5.91) or TNFi (OR 0.98, 0.68-1.40) there was no clear excess risk

Conclusion: In a large cohort, we did not detect a clear excess risk for offspring exposed in-utero to, vedolizumab or anti-TNF's, compared to unexposed patients there was a signal for more events with ustekinumab, but confidence intervals were wide. Ongoing caution, as well as more research on short and long-term effects, is warranted for biologics actively transported across the placenta

Disclosure of Interests: Jonah Gorodensky: None declared, Sasha Bernatsky: None declared, Waqqas Afif Consultant of: Abbvie, Amgen, Arena Pharmaceuticals, Dynacare, Janssen, Merck, Novartis, Pfizer, Sandoz, Takeda, Yvan St-Pierre: None declared, Kristian Filion: None declared, Evelyne Vinet: None declared

DOI: 10.1136/annrheumdis-2021-eular.691

\section{POS1421 \\ HIGHER SERUM URIC ACID IS ASSOCIATED WITH INCREASED RISK OF OBESITY IN CHINESE ADULTS: A LONGITUDINAL DATA ANALYSIS}

J. Zeng ${ }^{1}$, W. R. Lawrence ${ }^{2}$, J. Yang ${ }^{3} .{ }^{1}$ Department of Ultrasonography, Institute of Ultrasound in Musculoskeletal Sports Medicine, Guangdong Second Provincial General Hospital, Guangzhou, China, Department of 
Ultrasonography, Guangzhou, China; ${ }^{2}$ Department of Epidemiology and Biostatistics, School of Public Health, University at Albany, State University of New York, One University Place, Rensselaer, New York., Department of Epidemiology and Biostatistics, New York, United States of America; ${ }^{3}$ Institute for Environmental and Climate Research, Jinan University, Guangzhou, 511443, China, Jinan University, Guangzhou, China

Background: Hyperuricemia has been reported to be significantly associated with risk of obesity. However, previous studies on the association between serum uric acid (SUA) and body mass index (BMI) yielded conflicting results.

Objectives: The present study examined the relationship between SUA and obesity among Chinese adults.

Methods: Data were collected at Guangdong Second Provincial General Hospital in Guangzhou City, China between January 2010 and December 2018. Participants with $\geq 2$ medical checkup times were included in our analyses. Logistic regression model (LRM) was performed for data at baseline. For all participants, generalized estimation equation (GEE) model was used to assess the association between SUA and obesity, where the data were repeatedly measured over the nine-year study period. We calculated the cut-off values for SUA of obesity using the receiver operating characteristic curves $(\mathrm{ROC})$ technique.

Results: A total of 15,959 participants (10,023 males and 5,936females) were included in this study, with an average age of 37.38 years (SD: 13.27) and average SUA of $367.05 \mu \mathrm{mol} / \mathrm{L}$ (SD: 97.97) at baseline, respectively. Finally, 1078 participants developed obesity over the 9-year period. The prevalence of obesity was approximately $14.2 \%$ for high SUA level. In logistic regression analysis at baseline, we observed a positive association between SUA and risk of obesity: $\mathrm{OR}=1.84$ (95\% Cl: 1.77,1.90) for per-SD increase in SUA. Considering repeated measures over 9-year for all participants in the GEE model, the per-SD OR was 1.85 (95\% Cl:1.77,1.91) for SUA and the increased risk of obesity were greater for male $(O R=1.45)$ and elderly participants $(O R=1.01)$. The SUA cutoff points for risk of obesity using ROC curves were approximately consistent with the international standard.

Conclusion: Our study observed higher SUA level was associated with increased risk of obesity. More high-quality research is needed to further support these findings.

\section{REFERENCES:}

[1] Nagahama S, Kashino I, Hu H, Nanri A, Kurotani K, Kuwahara K et al. Haemoglobin A1c and hearing impairment: longitudinal analysis using a large occupational health check-up data of Japan. BMJ open 2018; 8(9): e023220.

[2] Kim YJ, Park H. Improving Prediction of High-Cost Health Care Users with Medical Check-Up Data. Big data 2019; 7(3): 163-175.

[3] Ishizaka N, Ishizaka Y, Toda A, Tani M, Koike K, Yamakado M et al. Changes in waist circumference and body mass index in relation to changes in serum uric acid in Japanese individuals. The Journal of rheumatology 2010; 37(2): 410-6.

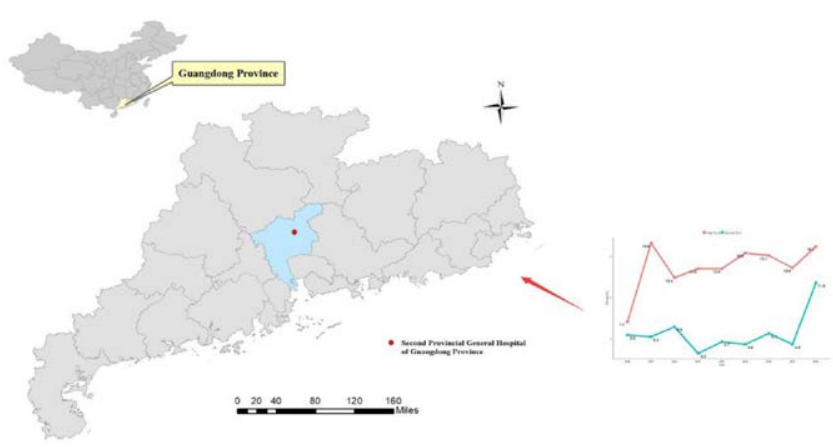

Figure 1. Location of Guangdong Second Provincial General Hospital (Guangzhou, Guangdong, China) and the prevalence of obesity by different years stratified by baseline SUA.

Disclosure of Interests: None declared

DOI: 10.1136/annrheumdis-2021-eular.752

\section{POS1422 CORRELATES OF TESTING POSITIVE FOR SARS-COV-2 IN PATIENTS WITH RHEUMATIC AD MUSCULOSKELETAL DISEASES}

N. Singh ${ }^{1,2}$, I. Huang ${ }^{2}$, M. Singleton ${ }^{2}$, A. Bays ${ }^{2}$, J. Sabo², S. Chung ${ }^{2}$, G. Gardner ${ }^{2}$, J. Schaeffer ${ }^{2}$, K. Wysham ${ }^{2}$, J. Andrews ${ }^{2}$, R. Patel ${ }^{2}$, J. Simard ${ }^{3}$, J. Liew ${ }^{4} .{ }^{1}$ University of Washington, Rheumatology, Bellevue; ${ }^{2} 1959$ NE Pacific St, Internal Medicine, Seattle, United States of America; ${ }^{3} 450$ Serra Mall, Internal Medicine, Stanford, United States of America; ${ }^{4} 233$ Bay State Rd, Internal Medicine, Boston, United States of America
Background: Many studies on COVID-19 outcomes in patients with RMD have either restricted to COVID positive RMD patients or compared them to the general clinic population as a comparator. Given heterogeneity in behaviors and risks, clinical characteristics associated with a positive diagnosis among patients with RMD seeking testing for Sars-CoV-2 remain less well studied.

Objectives: Among patients with RMD receiving a Sars-CoV-2 PCR test, we aimed to identify RMD-related factors associated with a positive test result.

Methods: Among patients seen at least once in the University of Washington (UW) rheumatology clinics between March 2018 to March 2020, we reviewed electronic medical records to identify patients undergoing Sars-CoV-2 PCR testing from March 1 through October 31, 2020. Patients with RMD were categorized into two groups: those who tested positive for Sars-CoV-2 and those who tested negative. We randomly selected patients from the negative group in a 2:1 ratio for further data abstraction. Student's $t$-test and Chi-squared tests were used to compare continuous and categorical variables, respectively, between the groups. To determine the correlates of testing positive for Sars-CoV-2, specifically RMD medication use and disease activity, we constructed different multivariable logistic regression models adjusted for age, sex, race/ethnicity, presence of comorbidities, body mass index, and smoking.

Results: A total of 2768 RMD patients underwent SARS-CoV-2 PCR testing within the UW system, of whom $43(1.5 \%)$ were positive at least once. Three patients with incomplete information were excluded. Patients who tested positive had higher prevalence of end stage renal disease (ESRD)/chronic kidney disease (CKD) $(24 \%$ versus $11 \%)$, had higher rates of active disease $(24 \%$ versus $20 \%$ ), were older ( $>55$ years) (mean age 57.3 versus 54.8 years), male ( $63 \%$ versus $55 \%$ ), non-white race/ethnicity (32\% versus $26 \%$ ), and higher prevalence of multiple comorbidities ( $42 \%$ versus $31 \%$ ) (Table 1 ). In the multivariable models, neither RMD medication use (versus no use, Table 1) nor high disease activity (vs low disease activity/remission) were statistically significantly associated with COVID-19 positivity. Among the 41 COVID-19 positive patients, a majority recovered without specific treatments, although approximately one third of the positive patients were hospitalized and three deaths were observed.

Conclusion: In this study, patients who tested positive did not differ in many ways from those who tested negative.

Table 1. Baseline characteristics of the patients prior to COVID testing

\begin{tabular}{|c|c|c|c|c|}
\hline Variables & $\begin{array}{c}\text { All } \\
(\mathrm{N}=126)\end{array}$ & $\begin{array}{l}\text { COVID } \\
\text { Positive } \\
\text { (N=41) }\end{array}$ & $\begin{array}{l}\text { COVID } \\
\text { Negative } \\
(\mathrm{N}=85)\end{array}$ & $\begin{array}{c}P \\
\text { value }\end{array}$ \\
\hline Age in years - mean (SD) & $55.6(15.3)$ & $57.3(16.3)$ & $54.8(14.9)$ & 0.40 \\
\hline Sex & & & & 0.39 \\
\hline Male & 73 (57.9) & $26(63.4)$ & $47(55.3)$ & \\
\hline Female & $53(42.1)$ & $15(36.6)$ & $38(44.7)$ & \\
\hline Race & & & & 0.39 \\
\hline White & 89 (71.2) & $26(63.4)$ & $63(74.1)$ & \\
\hline Other race & 35 (28.2) & $13(31.7)$ & $22(25.9)$ & \\
\hline Missing & $2(1.6)$ & $2(4.9)$ & $0(0.0)$ & \\
\hline Rheumatic disease & & & & 0.64 \\
\hline OA/Crystal/Fibromyalgia & $37(29.4)$ & $11(26.8)$ & $26(30.6)$ & \\
\hline $\mathrm{RA} / \mathrm{SpA}$ & $32(25.4)$ & $9(22.0)$ & $23(27.1)$ & \\
\hline All others & $57(45.2)$ & $21(51.2)$ & $36(42.3)$ & \\
\hline Rheumatic disease activity & & & & 0.57 \\
\hline Active & $27(21.4)$ & $10(24.4)$ & $17(20.0)$ & \\
\hline Not active & 99 (78.6) & 31 (75.6) & $68(80.0)$ & \\
\hline \multicolumn{5}{|l|}{ Co-morbidities } \\
\hline Diabetes mellitus (\%) & $25(19.8)$ & $9(22.0)$ & $16(18.8)$ & 0.68 \\
\hline Hypertension & $48(38.1)$ & $20(48.8)$ & $28(32.9)$ & 0.09 \\
\hline Cardiovascular disease & $23(18.3)$ & $9(22.0)$ & $14(16.5)$ & 0.46 \\
\hline Lung disease & 25 (19.8) & $10(24.4)$ & $15(17.7)$ & 0.37 \\
\hline Cancer & $10(7.9)$ & $3(7.3)$ & $7(8.2)$ & 0.86 \\
\hline ESRD/CKD & $19(15.1)$ & $10(24.4)$ & $9(10.6)$ & $0.04^{*}$ \\
\hline
\end{tabular}

BMI: Body mass index; SD: Standard deviation; OA: Osteoarthritis; Crystal: Crystalline diseases; RA: Rheumatoid arthritis; SpA: Spondyloarthritis

Acknowledgements: The work in this study was supported by grant UL1 TR002319 to Dr Singh from the Institute of Translational Health Sciences of the University of Washington.

Disclosure of Interests: None declared

DOI: 10.1136/annrheumdis-2021-eular.858

\section{POS1423 LIFESTYLE HABITS IN PATIENTS WITH RHEUMATOID ARTHRITIS - A CROSS SECTIONAL STUDY ON TWO SCANDINAVIAN COHORTS}

J. K. Karstensen $^{1,2,3}$, J. Primdahl ${ }^{1,2,4}$, M. Andersson ${ }^{5,6}$, J. Reffstrup Christensen ${ }^{7,8}$, A. Bremander $1,2,3,5,6 .{ }^{1}$ Department of Regional Health Research, University of Southern Denmark, Odense, Denmark; ${ }^{2}$ Danish Hospital for Rheumatic Diseases, University Hospital of Southern Denmark, Sønderborg, Denmark; ${ }^{3}$ The DANBIO Registry, Danish Hospital for Rheumatic Diseases, Sønderborg, Denmark; ${ }^{4}$ Hospital of Southern Jutland, University Hospital 